\title{
Superfluid-Insulator Transitions of the Fermi Gas with Near-Unitary Interactions in a Periodic Potential
}

\section{Citation}

Moon, Eun Gook, Predrag Nikolić, and Subir Sachdev. 2007. “Superfluid-Insulator Transitions of the Fermi Gas with Near-Unitary Interactions in a Periodic Potential." Physical Review Letters 99 (23). https://doi.org/10.1103/physrevlett.99.230403.

\section{Permanent link}

http://nrs.harvard.edu/urn-3:HUL.InstRepos:41417313

\section{Terms of Use}

This article was downloaded from Harvard University's DASH repository, and is made available under the terms and conditions applicable to Other Posted Material, as set forth at http:// nrs.harvard.edu/urn-3:HUL.InstRepos:dash.current.terms-of-use\#LAA

\section{Share Your Story}

The Harvard community has made this article openly available. Please share how this access benefits you. Submit a story. 


\title{
Superfluid-insulator transitions of the Fermi gas with near-unitary interactions in a periodic potential
}

\author{
Eun Gook Moon, Predrag Nikolić, and Subir Sachdev \\ Department of Physics, Harvard University, Cambridge MA 02138
}

(Dated: November 26, 2018)

\begin{abstract}
We consider spin- $1 / 2$ fermions of mass $m$ with interactions near the unitary limit. In an applied periodic potential of amplitude $V$ and period $a_{\mathrm{L}}$, and with a density of an even integer number of fermions per unit cell, there is a second-order quantum phase transition between superfluid and insulating ground states at a critical $V=V_{\mathrm{c}}$. We compute the universal ratio $V_{\mathrm{c}} m a_{\mathrm{L}}^{2} / \hbar^{2}$ at $N=\infty$ in a model with $\mathrm{Sp}(2 N)$ spin symmetry. The insulator interpolates between a band insulator of fermions and a Mott insulator of fermion pairs. We discuss implications for recent experiments.
\end{abstract}

An important milestone in the studies of ultracold atoms has been the observation of superfluidity in degenerate gases of fermionic atoms across a two-body Feshbach resonance [1, 2, 3, 4, 5]. As a function of the detuning, $\nu$, across the resonance, these systems interpolate from a Bose-Einstein condensate of diatomic molecules for large negative $\nu$, to a Bardeen-CooperSchrieffer (BCS) paired state of a Fermi liquid for large positive $\nu$. For $\nu \approx 0$, neither limiting description applies, and we have a superfluid state of fermions with interactions near the unitarity limit. As has been emphasized in recent work [6, 7, 8$]$ this entire crossover has striking universal aspects, with all physical properties determined only by $\nu$ and the density of the Fermi gas.

In a separate development, ultracold gases of bosonic atoms were placed in an optical lattice potential [9]. With increasing lattice depth, the bosons exhibited a superfluid-to-insulator quantum phase transition.

In the quest to realize strongly correlated quantum phases of interacting fermions, a recent experiment [10] has combined the techniques of the above experiment by studying fermions of mass $m$ with near unitary interactions in the presence of an optical lattice potential of period $a_{\mathrm{L}}$ and amplitude $V$. This paper shall demonstrate that the universality arguments can be extended to include the periodic potential after including a single energy scale associated with $V$. In particular, we find a universal phase diagram shown in Fig 1 below. This phase diagram has superfluid-insulator quantum phase transitions with a density of an even integer number $\left(n_{\mathrm{F}}\right)$ of fermions per unit cell at critical amplitude $V=V_{\text {c }}$ which obeys

$$
V_{\mathrm{c}}=\frac{\pi^{2} \hbar^{2}}{4 m a_{\mathrm{L}}^{2}} F_{n_{\mathrm{F}}}\left(a_{\mathrm{L}} \nu\right)
$$

where $F_{n_{\mathrm{F}}}$ is a universal function of $a_{\mathrm{L}} \nu$ dependent only on the even integer $n_{\mathrm{F}}$. The transition at unitarity occurs at $F_{n_{\mathrm{F}}}(0)$. We will show that $F_{n_{\mathrm{F}}}$ can be determined in a $1 / N$ expansion in a model with $\operatorname{Sp}(2 N)$ spin symmetry. Explicit numerical results for the universal phase diagram and the function $F_{n_{\mathrm{F}}}$ will be presented below at $N=\infty$.

An important aspect of the physics accounted for by our analysis is that the insulator near the critical point is a novel quantum state which is neither a band insulator of fermions, nor a Mott insulator of bosonic fermion pairs. Instead, it is in a interesting intermediate regime in which multiple single-particle bands are occupied. Previous computations of ultra-cold atoms in optical lattices have relied on effective tight-binding models [1, 12, 13, 14], but such approaches are not expected to be quantitatively accurate near the transition to the superfluid. A recent computation [15] uses a method related to ours, but does not account for the off-diagonal couplings between different reciprocal lattice vectors in Eq. (6) below; these are essential for a proper result, and make the computation much more demanding. Our $1 / N$ expansion is also able to quantitatively account for the strong interactions between the fermions in the multiple bands. One consequence of the many occupied bands is that the computational requirements are demanding even at the leading $N=\infty$ level. So our present numerical results will be limited to $N=\infty$ although we will set up a formalism that allows computations to all orders in $1 / N$. It is also worth noting that previous studies of ground state properties [7] found that $1 / N$ corrections were quite small in the unitarity limit, $\nu \approx 0$.

We consider $2 N$ species of fermionic atoms $\psi_{i \sigma}(i=$ $1 \ldots N, \sigma \in\{\uparrow, \downarrow\})$ coupled to a single field $\Phi$ of s-wave Cooper pairs, or molecules. This is an $\operatorname{Sp}(2 N)$ generalization of the popular "two-channel" model, and the physical case $N=1$ can be accessed in $1 / N$ expansions [6, 7]. The atoms experience an optical lattice potential $V(\boldsymbol{r})$; we choose a simple cubic latice potential

$$
V(\boldsymbol{r})=V\left[\cos ^{2}\left(\frac{\pi x}{a_{\mathrm{L}}}\right)+\cos ^{2}\left(\frac{\pi y}{a_{\mathrm{L}}}\right)+\cos ^{2}\left(\frac{\pi z}{a_{\mathrm{L}}}\right)\right],
$$

and our computations have simple generalization to other lattice structures. The density of the fermions is controlled by chemical potential $\mu$ and we assume that spin polarization is zero. The imaginary-time action of this many-body system, which includes all terms for a description of the universal physics in the vicinity of a two- 
body Feshbach resonance is:

$$
\begin{aligned}
\mathcal{S}= & \int \mathrm{d} \tau \mathrm{d}^{3} r\left[\psi_{i \sigma}^{\dagger}\left(\frac{\partial}{\partial \tau}-\frac{\nabla^{2}}{2 m}-\mu+V(\boldsymbol{r})\right) \psi_{i \sigma}\right. \\
& \left.+\Phi^{\dagger} \psi_{i \downarrow} \psi_{i \uparrow}+\Phi \psi_{i \uparrow}^{\dagger} \psi_{i \downarrow}^{\dagger}\right]+N \frac{m \nu}{4 \pi} \int \mathrm{d} \tau \mathrm{d}^{3} r \Phi^{\dagger} \Phi
\end{aligned}
$$

Note that the Cooper pair field $\Phi$ is actually a HubbardStratonovich field that decouples the fermion interaction terms, and hence does not have a bare dispersion, or Berry's phase. Thanks to this, we have conveniently rescaled the $\Phi$ field to absorb in it the interaction coupling. Among the relevant operators is detuning $\nu$ from the Feshbach resonance in the absence of the lattice, and the co-efficient that contains it is fixed by relating the scattering matrix of this theory to the scattering length $a=-1 / \nu$ for $V(\boldsymbol{r})=0$.

Our primary result is that the above model has a universal phase diagram as a function of $\mu / E_{\mathrm{r}}, E_{\mathrm{r}} / V$, and $\nu a_{\mathrm{L}}$, where $E_{\mathrm{r}}=\pi^{2} \hbar^{2} /\left(4 m a_{\mathrm{L}}^{2}\right)$ is the molecular recoil energy. The phase boundaries are shown in Fig. 1 as a function of the first two parameters for different values of $\nu a_{\mathrm{L}}$.

We will explore the superfluid phase boundary by focusing on the superfluid order parameter. We integrate out the fermion fields $\psi_{i \sigma}$ and obtain the effective action of the Cooper pair field $\Phi$, which can be expressed using Feynman diagrams:

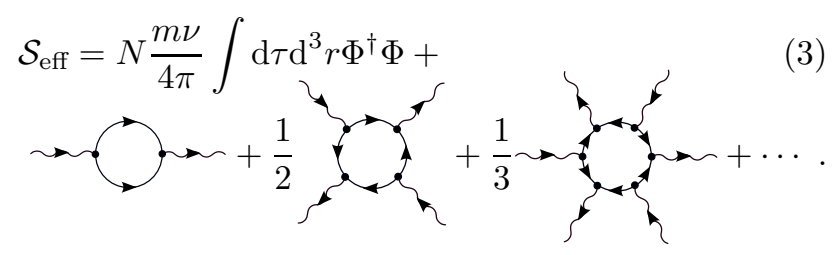

Each fermion loop (straight lines) contributes a factor of $N$, so that $N$ appears as an overall factor in the effective action. Every external wavy line represents the pair field $\Phi$ or $\Phi^{\dagger}$, depending on the direction of arrow with respect to vertex, and every vertex is associated with a point in space and time. In the present problem, the optical lattice potential $V(\boldsymbol{r})$ breaks translation symmetry, so that momentum is not a good quantum number. Instead, according to the Bloch's theorem, quantum numbers are band index $\boldsymbol{n}$ and crystal momentum $\boldsymbol{k}$ which takes values within the first Brillouin zone (BZ). Assuming a Bravais optical lattice with reciprocal vectors $\boldsymbol{G}$, the bare fermion propagator is given by:

$$
\begin{aligned}
G_{0}(1,2) & =\sum_{\boldsymbol{n}} T \sum_{\omega} \int_{\mathrm{BZ}} \frac{\mathrm{d}^{3} k}{(2 \pi)^{3}} \sum_{\boldsymbol{G}_{1}, \boldsymbol{G}_{2}} \frac{\psi_{\boldsymbol{n}, \boldsymbol{k} ; \boldsymbol{G}_{1}}^{*} \psi_{\boldsymbol{n}, \boldsymbol{k} ; \boldsymbol{G}_{2}}}{-i \omega+\epsilon_{\boldsymbol{n}, \boldsymbol{k}}} \\
& \times e^{i\left(\boldsymbol{k}\left(\boldsymbol{r}_{2}-\boldsymbol{r}_{1}\right)-\omega\left(\tau_{2}-\tau_{1}\right)\right)} e^{i\left(\boldsymbol{G}_{2} \boldsymbol{r}_{2}-\boldsymbol{G}_{1} \boldsymbol{r}_{1}\right)}
\end{aligned}
$$

where the Fourier components of the Bloch wavefunctions $\psi_{\boldsymbol{n}, \boldsymbol{k} ; \boldsymbol{G}}$ and energies $\epsilon_{\boldsymbol{n}, \boldsymbol{k}}$ are obtained from the Fourier transformed Schrödinger equation:

$$
\begin{aligned}
\sum_{\boldsymbol{G}^{\prime}} & {\left[\left(\frac{(\boldsymbol{k}+\boldsymbol{G})^{2}}{2 m}-\mu\right) \delta_{\boldsymbol{G}, \boldsymbol{G}^{\prime}}+V_{\boldsymbol{G}-\boldsymbol{G}^{\prime}}\right] \psi_{\boldsymbol{n}, \boldsymbol{k} ; \boldsymbol{G}^{\prime}}=} \\
& =\epsilon_{\boldsymbol{n}, \boldsymbol{k}} \psi_{\boldsymbol{n}, \boldsymbol{k} ; \boldsymbol{G}} \quad(\forall \boldsymbol{k}, \boldsymbol{G}) .
\end{aligned}
$$

Solving this set of equations numerically requires imposing a cut-off energy $\Lambda$ that limits the reciprocal vectors kept in calculations: $\boldsymbol{G}^{2}<2 m \Lambda$. For our $V(\boldsymbol{r})$ above, the only non-zero Fourier components are $V_{0}=3 V / 2$, $V_{ \pm 2 \pi \hat{\boldsymbol{x}} / a_{\mathrm{L}}}=V_{ \pm 2 \pi \hat{\boldsymbol{y}} / a_{\mathrm{L}}}=V_{ \pm 2 \pi \hat{\boldsymbol{z}} / a_{\mathrm{L}}}=V / 4$.

The presence of superfluidity can be described by a superfluid order parameter, which due to the optical lattice may have Fourier components $\Phi_{G}$ at various reciprocal lattice vectors $\boldsymbol{G}$. Fluctuations $\delta \Phi(\boldsymbol{r})$ of the Cooper pair field are added to the order parameter, and the total boson field $\Phi(\boldsymbol{r})=\sum_{\boldsymbol{G}} \Phi_{\boldsymbol{G}} e^{i \boldsymbol{G r}}+\delta \Phi(\boldsymbol{r})$ is represented by the wavy lines in (3). For large $N$ the action $\mathcal{S}_{\text {eff }} \propto N$ becomes large, so that fluctuations $\delta \Phi$ are suppressed; the mean-field theory becomes exact in the limit $N \rightarrow \infty$. Integrating out $\delta \Phi$ gives rise to corrections of the order $1 / N$ to the mean-field results. This follows from diagrammatic perturbation theory performed in the effective action, where the first diagram in (3), together with the detuning term, defines a 'bare' propagator of the $\Phi$ fields, while all other diagrams define new vertices of the $\Phi$ fields. Even though the new vertices are proportional to $N$, the 'bare' propagator is proportional to $1 / N$ and hence yields perturbative expansions of thermodynamic functions in powers of $1 / N$.

As in the bosonic case, we expect a second order superfluid-insulator transition. Near such a transition, the action terms quadratic in order parameter determine the state, in analogy to a simple uniform $\Phi^{4}$ LandauGinzburg theory of bosons. Neglecting the fluctuations $\delta \Phi(\boldsymbol{r})$ in the $N \rightarrow \infty$ limit, the free energy density $\mathcal{F}$ is just the effective saddle-point action divided by volume $\mathcal{V}$ and $\beta=1 / T$ :

$$
\frac{\mathcal{F}}{N}=\frac{\mathcal{S}_{\mathrm{eff}}}{N \beta \mathcal{V}}=\sum_{\boldsymbol{G}, \boldsymbol{G}^{\prime}} K_{\boldsymbol{G}, \boldsymbol{G}^{\prime}}^{(2)} \Phi_{\boldsymbol{G}}^{\dagger} \Phi_{\boldsymbol{G}^{\prime}}+\mathcal{O}\left(\Phi^{4}\right)
$$

The quadratic couplings $K_{\boldsymbol{G}, \boldsymbol{G}^{\prime}}^{(2)}$ can be represented as a matrix whose components are indexed by the reciprocal lattice vectors $\boldsymbol{G}$. If this matrix has only positive eigenvalues, the free energy ([6) is minimized by $(\forall \boldsymbol{G}) \Phi_{\boldsymbol{G}}=0$ indicating an insulating $(T=0)$ or normal $(T>0)$ phase. Otherwise, the minimum is obtained at $(\exists \boldsymbol{G}) \Phi_{\boldsymbol{G}} \neq 0$, and the established phase is superfluid. The role of $\mathcal{O}\left(\Phi^{4}\right)$ terms near a second order phase transition is only to stabilize the theory.

A naive derivation of $K_{\boldsymbol{G}, \boldsymbol{G}^{\prime}}^{(2)}$ from the first Feynman diagram in (3) produces an ultra-violet divergent expression. This divergence stems from the naive continuum form of the bare field theory, and must be renormalized away by absorbing it into finite physically measurable 
renormalized quantities. One step toward this goal has already been taken by absorbing any bare molecule mass (a part of the bare molecule dispersion omitted from (2)) into detuning $\nu$, which is measurable and fixed in the effective field theory simply by the properties of scattering matrix. The second step is to remove the remaining unphysical divergent part by dimensional regularization, which at large momenta $\boldsymbol{k}+\boldsymbol{G}$ is carried out just like in a system without the optical lattice[6]. The regularized expression is:

$$
\begin{aligned}
& K_{\boldsymbol{G}, \boldsymbol{G}^{\prime}}^{(2)}=\frac{m \nu}{4 \pi} \delta_{\boldsymbol{G}, \boldsymbol{G}^{\prime}}+\int_{\mathrm{BZ}} \frac{\mathrm{d}^{3} k}{(2 \pi)^{3}}\left\{\sum_{\boldsymbol{G}^{\prime \prime}} \frac{m \delta_{\boldsymbol{G}, \boldsymbol{G}^{\prime}}}{\left(\boldsymbol{k}+\boldsymbol{G}^{\prime \prime}\right)^{2}}-\right. \\
& \sum_{\boldsymbol{n}_{1}, \boldsymbol{n}_{2}} \sum_{\boldsymbol{G}_{1}, \boldsymbol{G}_{2}}\left[1-f\left(\epsilon_{\boldsymbol{n}_{1}, \boldsymbol{k}}\right)-f\left(\epsilon_{\boldsymbol{n}_{2},-\boldsymbol{k}}\right)\right] \times \\
& \left.\frac{\left(\psi_{\boldsymbol{n}_{1}, \boldsymbol{k} ; \boldsymbol{G}_{1}}^{*} \psi_{\boldsymbol{n}_{2},-\boldsymbol{k} ; \boldsymbol{G}^{\prime}-\boldsymbol{G}_{1}}^{*}\right)\left(\psi_{\boldsymbol{n}_{1}, \boldsymbol{k} ; \boldsymbol{G}_{2}} \psi_{\boldsymbol{n}_{2},-\boldsymbol{k} ; \boldsymbol{G}-\boldsymbol{G}_{2}}\right)}{\epsilon_{\boldsymbol{n}_{1}, \boldsymbol{k}}+\epsilon_{\boldsymbol{n}_{2},-\boldsymbol{k}}}\right\},
\end{aligned}
$$

where $f(x)=\left(1+e^{x / T}\right)^{-1}$ is the Fermi-Dirac distribution function.

In the following, we numerically compute the matrix $K^{(2)}$ for reciprocal lattice vectors $|\boldsymbol{G}|<\sqrt{2 m \Lambda^{\prime}}$, where $\Lambda^{\prime} \leq \Lambda$, in the limit $N \rightarrow \infty$. In practice, it is sufficient to choose a very small $\Lambda^{\prime}\left(|\boldsymbol{G}| \leq 2 \pi a_{\mathrm{L}}^{-1}\right)$, as long as $\Lambda \gg$ $V$. By mapping dependence of the smallest eigenvalue $\Gamma^{(2)}$ of the matrix $K^{(2)}$ on chemical potential $\mu$, lattice amplitude $V$ and detuning $\nu$, we find the second order phase boundary between superfluid and insulating phases by the condition

$$
\Gamma^{(2)}=0
$$

In Fig 1 we show a contour plot of the phase boundary as a function of chemical potential and inverse lattice amplitude at $T=0$ and $N=\infty$. This choice of plot axes was made in order to obtain resemblance to the wellknown phase diagram [16] of a superfluid to Mott insulator transition; while chemical potential directly controls density, inverse lattice amplitude is correlated with hopping strength $t$ of an effective tight-binding model. Indeed, insulating regions are dome-shaped and correspond to integer fillings of optical lattice sites with bosons (Cooper pairs). The character of an insulator depends on detuning $\nu$ from the Feshbach resonance. On the BCS side of the resonance, $\nu>0$, the insulating domes become larger, and converge toward the fermion band-gap boundaries as $\nu$ is increased. The system then behaves as a typical band-insulator (weakly paired fermions are insulating due to a filled band). On the other hand, in the BEC limit $\nu<0$ the domes become 'smaller', resembling a Mott insulator. When molecules are tightly bound, filling up a fermion band is not sufficient to destroy superfluidity, but repulsion between molecules needs to step in. Note, however, that true Mott insulating phases, with

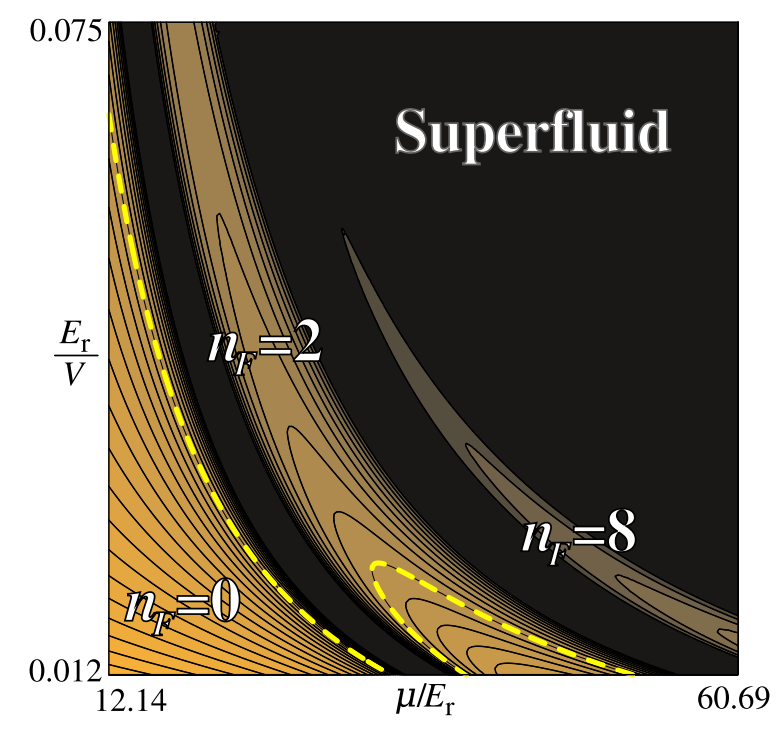

FIG. 1: A contour plot of the superfluid-insulator phase boundaries at $T=0$ and $N=\infty$. Contours, dependent on detuning $\nu$ from the Feshbach resonance, separate superfluid regions surrounding the fermion bands (black) from insulating regions in the band structure gaps. The dashed yellow contours correspond to the transition at the resonance $\nu=0$, while the spacing between contours is $\Delta \nu=a_{\mathrm{L}}^{-1}$, where $a_{\mathrm{L}}$ is the optical lattice spacing; contours move upward and toward the band edges as $\nu$ grows. Insulators are labeled by the closest even integer to the average filling of the optical lattice by atoms in the grand-canonical ensemble. The reference energy scale is molecule recoil energy $E_{\mathrm{r}}=\pi^{2} \hbar^{2} /\left(4 m a_{\mathrm{L}}^{2}\right)$. Accuracy is smaller than that in Fig 2 because calculating at many points in reasonable time required a small cut-off $\Lambda=3 \times\left(2 \pi a_{\mathrm{L}}^{-1}\right)$.

an arbitrary integer lattice filling by molecules, cannot be found without including $1 / N$ corrections.

It is also important to consider the phase diagram as a function of particle density, rather than chemical potential. The Mott insulating lobes in Fig. 1 all have a density of an even integer number, $n_{\mathrm{F}}$, of fermions per unit cell. We therefore fix the density at $n_{\mathrm{F}}$ and study the transition from the insulator to the superfluid. By generalizing the argument made for the bosonic case, this $T=0$ transition occurs at the point where we satisfy the condition (8) along with

$$
\frac{\partial \Gamma^{(2)}}{\partial \mu}=0 .
$$

The two conditions (8) and (9) determine an isolated point in the phase diagram of Fig. 1 for each $n_{\mathrm{F}}$, and the location of these points then immediately yields Eq. (11). In Fig. 2 we plot the universal function $F_{n_{F}}\left(\nu a_{\mathrm{L}}\right)$ for $n_{\mathrm{F}}=2$ and $N=\infty$ (calculations were performed with large cut-off $\left.\Lambda \leq 10 \times\left(2 \pi a_{\mathrm{L}}^{-1}\right)\right)$.

The obtained values of $V_{\mathrm{c}}=F_{n_{\mathrm{F}}}\left(a_{\mathrm{L}} \nu\right) E_{\mathrm{r}}$ are larger than those reported in the experiment, $V_{\mathrm{c}} \approx 6 E_{\mathrm{r}}[10]$. 


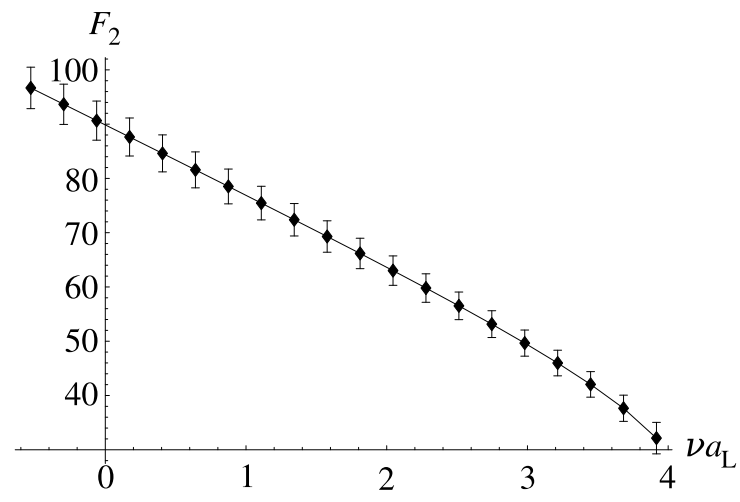

FIG. 2: Universal function $F_{n_{\mathrm{F}}}\left(\nu a_{\mathrm{L}}\right)$ in (1) at the superfluidinsulator transition for the first fermion band completely filled, $n_{\mathrm{F}}=2(T=0, N=\infty)$.

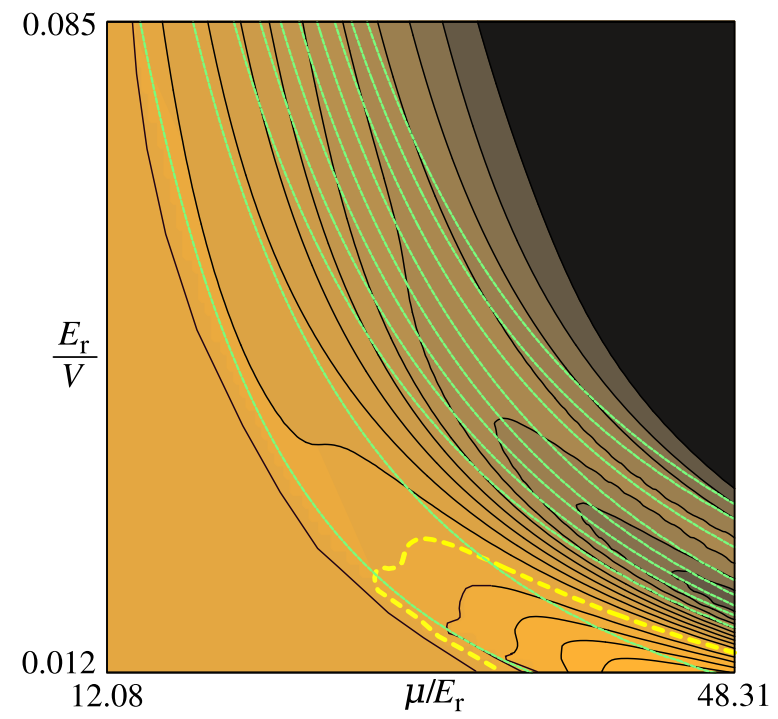

FIG. 3: A contour plot of the superfluid-normal phase boundary at $T \approx 1.8 E_{\mathrm{r}}$ and $N=\infty$. This temperature is $T \approx 0.6 E_{\mathrm{f}}$, where $E_{\mathrm{f}}=\hbar^{2}\left(6 \pi a_{\mathrm{L}}^{3}\right)^{2 / 3} /(2 m)$ is the Fermi energy of a free fermion gas at the same density as the fermion gas in the lattice with two particles per site. The black and yellow contours are contours of constant detuning, as in Fig 1 The green lines are normal-phase constant density lines, starting at $n_{\mathrm{F}}=1$ at the left-bottom, displaced by $\Delta n_{\mathrm{F}}=1$. The superconductornormal phase transition can occur at any density or detuning, at the intersection of the corresponding contour lines. Note that due to a small cut-off used in plotting this diagram, one should not directly compare it with Fig. 2 - the purpose is only to illustrate the shape of phase boundaries.

However, unlike our computation so far, the experiment is at a non-zero temperature (not known very accurately in the presence of the optical lattice), and thermal fluctuations should decrease the value of $V_{c}$. We extended our results to a range of $T>0$ as shown in Fig. 3. The character of the transition at which superconductivity is lost changes qualitatively at $T>0$. At $T=0$, the insulator must have a density $n_{F}=$ even integer, and so a superconductor-insulator transition can only occur at such values of $n_{F}$; this was rationale behind the additional constraint in Eq. (9). However, at the $T>0$, the transition is more properly a superconductor-normal transition, and the normal state can occur at any density. In Fig 3 we show a contour plot of the boundaries between superfluid and normal phases at finite temperatures $(N=\infty)$. As temperature is increased, the nonsuperfluid domes gradually expand. The contours corresponding to larger values of $\nu$ (BCS-limit) are more affected by thermal fluctuations than those corresponding to smaller values of $\nu$ (BEC-limit). Since at $T>0$ the normal regions can occur when the chemical potential is not in a band gap, the normal regions corresponding to different average lattice fillings can merge when the $\nu$-dependent effect of fluctuations is large enough; when this happens at a particular $\nu$, the appropriate contour stretches all the way from the bottom to the top of the diagram, instead of being dome-shaped. We expect that including molecule fluctuations would increase these effects even further. Now, if an experiment is performed at a fixed density (green lines in Fig. 33), the phase transition to a normal phase at unitarity (dashed line) can occur at a smaller lattice depth $V_{\mathrm{c}}$ than at $T=0$. The reduction of $V_{\mathrm{c}}$ can be particularly dramatic if the transition is observed in a region where the $T=0$ non-superfluid regions have merged due to thermal fluctuations. This can even occur at temperatures small compared to the Fermi energy. We suspect this effect is the primary reason for the discrepancy between the $T>0$ experiments and our $T=0$ results.

We thank S. Diehl for useful discussions, and J. K. Chin for helping us understand better the experiment [10]. This research was supported by the NSF under grant DMR-0537077. E. G. Moon is also supported in part by the Samsung Scholarship.

[1] C. A. Regal, M. Greiner, and D. S. Jin, Phys. Rev. Lett. 92, 040403 (2004).

[2] M. W. Zwierlein, C. A. Stan, C. H. Schunck, S. M. F. Raupach, A. J. Kerman, and W. Ketterle, Phys. Rev. Lett. 92, 120403 (2004).

[3] C. Chin, M. Bartenstein, A. Altmeyer, S. Riedl, S. Jochim, J. H. Denschlag, and R. Grimm, Science 305 1128 (2004).

[4] M. W. Zwierlein, J. R. Abo-Shaeer, A. Schirotzek, C. H. Schunck, and W. Ketterle, Nature 435, 1047 (2005).

[5] J. Stewart, J. P. Gaebler, C. A. Regal, and D. S. Jin, Phys. Rev. Lett. 97, 220406 (2006).

[6] P. Nikolić and S. Sachdev, Phys. Rev. A 75, 033608 (2007).

[7] M. Y. Veillette, D. E. Sheehy, and L. Radzihovsky, Phys. Rev. A 75, 043614 (2007).

[8] S. Diehl and C. Wetterich, Phys. Rev. A 73, 033615 
(2006); S. Diehl, H. Gies, J. M. Pawlowski, C. Wetterich, cond-mat/0703366 (2007)

[9] M. Greiner, O. Mandel, T. Esslinger, T. W. Hänsch, I. Bloch, Nature 415, 39 (2002).

[10] J. K. Chin, D. E. Miller, Y. Liu, C. Stan, W. Setiawan, C. Sanner, K. Xu, W. Ketterle, Nature 443, 961 (2006).

[11] L.-M. Duan, Phys. Rev. Lett. 95, 243202 (2005).

[12] K. B. Gubbels, D. B. M. Dickerscheid, and H. T. C. Stoof, New J. Phys. 8, 151 (2006).
[13] A. O. Koetsier, D. B. M. Dickerscheid, and H. T. C. Stoof, Phys. Rev. A 74, 033621 (2006).

[14] T. K. Koponen, T. Paananen, J.-P. Martikainen, and P. Torma, cond-mat/0701484

[15] H. Zhai, T.-L. Ho, cond-mat/0704.2957 (2007).

[16] M. P. A. Fisher, P. B. Weichmann, G. Grinstein, and D. S. Fisher, Phys. Rev. B 40, 546 (1989). 